\title{
A Tapered Whisker-Based Physical Reservoir Computing System for Mobile Robot Terrain Identification in Unstructured Environments
}

\author{
Zhenhua Yu ${ }^{1}$, Shehara Perera ${ }^{1}$, Helmut Hauser ${ }^{2}$, Peter R.N. Childs ${ }^{1}$,Thrishantha Nanayakkara ${ }^{1}$
}

\begin{abstract}
In this letter, we present for the first time the use of tapered whisker-based reservoir computing (TWRC) system mounted on a mobile robot for terrain classification and roughness estimation of unknown terrain. Hall effect sensors captured the oscillations at different locations along a tapered spring that served as a reservoir to map time-domain vibrations signals caused by the interaction perturbations from the ground to frequency domain features directly. Three hall sensors are used to measure the whisker reservoir outputs and these temporal signals could be processed efficiently by the proposed TWRC system which can provide morphological computation power for data processing and reduce the model training cost compared to the convolutional neural network $(\mathrm{CNN})$ approaches. To predict the unknown terrain properties, an extended TWRC method including a novel detector is proposed based on the Mahalanobis distance in the Eigen space, which has been experimentally demonstrated to be feasible and sufficiently accurate. We achieved a prediction success rate of $94.3 \%$ for six terrain surface classification experiments and $\mathbf{8 8 . 7 \%}$ for roughness estimation of the unknown terrain surface.
\end{abstract}

Index Terms-Robotic whiskers, Reservoir computing, Terrain classification, roughness estimation.

\section{INTRODUCTION}

$\mathbf{M}$ OBILE robots performing tasks in unknown environments need to traverse a variety of complex terrains, and they must be able to reliably and quickly identify and characterize these terrains to avoid getting into potentially challenging or catastrophic circumstances [1]. This is a challenging research problem because the visual appearance of outdoor terrain changes due to aspects such as weather, precipitation, light intensity and smoke, as well surface properties that can change due to dirt, water and snow.

\footnotetext{
*Manuscript received: September 9, 2021; Revised: November 9, 2021; Accepted: January 7, 2022.

This paper was recommended for publication by Editor Liu. Xinyu upon evaluation of the Associate Editor and Reviewers' comments. This work was partly supported by EU Horizon 2020 research and innovation programme under grant agreements 101016970 (Natural Intelligence for Robotic Monitoring of Habitats), UK Engineering and Physical Sciences Research Council (EPSRC) RoboPatient project under Grant EP/T00603X/1, and $821201 \mathrm{Cir}-$ cular Construction in Regenerative Cities (CIRCuIT). Corresponding author: Zhenhua Yu

${ }^{1}$ Zhenhua Yu, Shehara Perera, P.Childs, and T.Nanayakkara are with Dyson School of Design Engineering, Imperial College London, SW7 2DB, London, UK (e-mail:z.yu18@imperial.ac.uk, u.perera@imperial.ac.uk, p.childs@imperial.ac.uk, t.nanayakkara@imperial.ac.uk)

2 Helmut Hauser is with the Department of Engineering Mathematics, University of Bristol, Bristol BS8 1TH,U.K,and also with the SoftLab, Bristol Robotics Laboratory, U.K.(e-mail:helmut.hauser@bristol.ac.uk)

Digital Object Identifier (DOI): see top of this page.
}

These factors have motivated a variety of terrain identification research based on various sensors, using vision [2], lidar [3], audio [4], inertial measurement units (IMU) [5], tactile sensors [6], and multi-modal fusion [7]. Among the various modalities, vision-based terrain identification methods combined with deep learning have demonstrated extraordinary advantages and have led to significant technological developments [8]. For contact-sensor-based schemes, IMU [9] or tactile sensors [10] are investigated by using the direct contact information of the ground properties through application of convolutional neural network $(\mathrm{CNN})$ methods. Inspired by animals such as rats and sea lions which use whiskers to navigate in the dark and perceive environmental information and features without vision [11], artificial whiskers sensors have been designed and constructed for robot navigation [12], terrain identification [13], object detection [14], size measurement [15], shape recognition [16], and surface information identification [17].

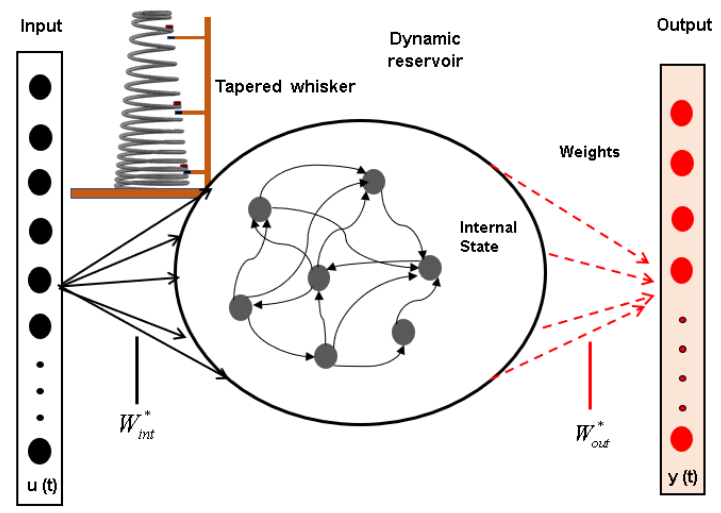

Fig. 1. Schematic of a whisker-based reservoir computing system, showing the internal reservoir and a readout function. The tapered shape causes different vibration frequency components to localise along the whisker when the tip is rubbed against a surface or the base is vibrated with the tip end is not in contact with any object. The nonlinear tapered whisker sensor serve as the reservoir, and only the weight matrix $W_{\text {out }}$ needs to be trained. There can be any number of readouts from the whisker. In this particular application of terrain classification, we use only 3 readouts for sufficient accuracy.

However, most of the above state-of-the-art conventional neural network-based methods require large training data samples, and these training data are often difficult to collect, especially in unknown environments because they demands manually assignments of the labels to the training dataset. Moreover, these approaches require huge computing resources 
for online training and recognition, and the performance tends to degrade once there are outlier data that do not exist in the prior training dataset. As a result, it is difficult to quickly and cost-efficiently predict the surface characteristics of an emerging unknown terrain, especially, for vehicle-mounted IMUs and vision methods in complex extreme environments, which may not be feasible in practical robotic applications in unstructured environments.

An alternative, recently emerging, approach is to use physical reservoir computing [18]. This exploits the complex dynamics of physical bodies as a computational resource and therefore can significantly reduce the computational power needed. The approach is based on a machine learning approach called reservoir computing [19], which can be used to learn dynamic input output relationships. At its centre is a highdimensional, nonlinear dynamical system (i.e., the reservoir) which maps nonlinearily any input signals into its high dimensional state space and at the same time integrates temporal information with a leaky memory, see Fig.1. If the dynamics of the reservoir are sufficiently complex, then, adding a linear readout is sufficient to emulate complex nonlinear dynamical systems [18]. This means, with the help of the nonlinear dynamics of the reservoir, learning complex computations can be reduced to finding a set of linear output weights by using simple linear regression. While reservoir computing is an abstract machine learning method, physical reservoir computing uses real physical systems as reservoirs. This includes nonlinear effects in lasers, networks of memristors, compliant body parts of robots and may others [20] [21].

In our case, we use a tapered spring as the reservoir (see Fig.1) mounted on a mobile robot. The idea is that movements of the robot on different types of rough terrain will result in different vibration behaviours of the spring. The readout from the reservoir is implemented by three magnets located at the bottom, middle and top of the spring. Corresponding Hall effect sensor obtains vibration readings. Specifically, to learn a corresponding classification (i.e., classify terrain and roughness based on the sensory signal) logistic regression will be used. Note that in previous research, Hauser et al. [22] has demonstrated that a network of compliant mass-spring systems can be used to emulate complex nonlinear systems (e.g. controller, filters, limit cycles, etc.). In this paper, we only use one spring, but being tapered, it allows us to access different frequency components simultaneously.

To our knowledge, this is the first time a tapered whiskerbased reservoir computing system is used for terrain classification and roughness estimation of unknown terrain for a mobile robot (Fig.2). Reservoir computing is deployed here by using the whisker sensor to project features from the temporal vibration data directly for logistic regression training, which can provide embodied computing input for data processing and reduce computational cost of model training. This paper firstly shows that the steady state response of nonlinear vibration dynamics of whisker reservoir outputs can be used to classify terrain textures even on flat terrain and estimate the roughness of the unknown terrains, which significantly improves the realtime properties of the terrain identification.

The remainder of this paper is organized as follows: Section
II introduces the design and construction of tapered springbased whisker reservoir computing system and workflow. The reservoir computing based on whisker sensor vibration characteristics and readout training are studied in Section III. Section IV reports the whisker vibration data collection and reservoir feature extraction as well as algorithm framework for surface roughness estimation of unknown terrains. Section $\mathrm{V}$ presents the results and analysis of terrain identification experiments and prediction of the unknown terrain's roughness based on reservoir computing. We also provide comparative experimental results to demonstrate that it is not possible to do classification work if linear IMU data, e.g., acceleration, are used directly. Finally, Section VI concludes this paper's contribution and discusses future work.

\section{Reservoir Computing System Design}

\section{A. Conical Spring-based Whisker Sensor Design}

In this paper, we use the phenomenon of vibration frequency separation along the axis of a tapered spring vertically mounted on a mobile robot as part of reservoir computer to classify terrain types. Fig.2 illustrates the proposed nonlinear whiskered sensor for terrain identification. The body of the physical reservoir was made of a high-carbon conical nonlinear spring, with a free length: $100 \mathrm{~mm}$, wire diameter: $1 \mathrm{~mm}$, bottom diameter: $20 \mathrm{~mm}$, and tip diameter: $10 \mathrm{~mm}$ respectively. Three neodymium permanent magnets are embedded in the bottom, middle, and top of the conical spring beam. Consequently, three SS49E linear Hall sensors are orthogonally mounted under the neodymium permanent magnets by the cantilever beam to measure the osculation induced by terrain. The cantilever used to support the Hall sensors is made of rigid acrylic pillars to keep the relative positions of three Hall sensors remaining relatively constant when the robot moving.

The saturation of the whisker sensor reading depends on the Hall sensor, which has a reading range from $1 \mathrm{~V}$ to $4 \mathrm{~V}$ with magnetic fields from -1000 Gauss to 1000 Gauss. The sensitivity of the SS49E Hall sensor is $1.4 \mathrm{mV} /$ Gauss, which enables capture of extremely weak magnetic field vibrations and ability to distinguish quite similar terrains or surfaces.

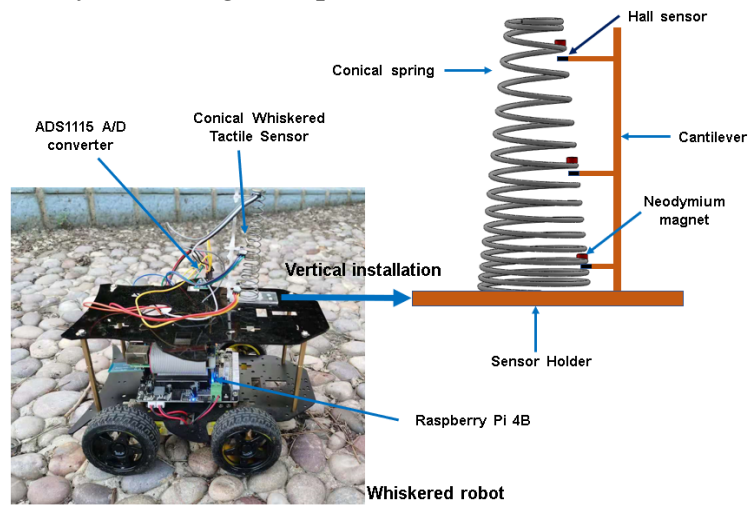

Fig. 2. The whiskered mobile robot equipped with a bioinspired tapered whisker-based reservoir computing system. The tapered spring-based whisker sensor is vertically mounted on the front of the mobile robot to collect the nonlinear vibration directly.

The whisker sensor is installed orthogonally in front of the wheeled robot because when the robot travels through 
different terrains, the front wheels of the robot will receive the external terrain excitation first, which helps the robot to recognize the new terrain information earlier to avoid catastrophic consequences, as shown in Fig.2. When the robot traverses different terrains in steady-state motion, the external vibration will act vertically on the conical whiskered spring shaft, which will cause nonlinear perpendicular displacement along the tapered spring axis. The shaft deformation also induces the same vibrations on the magnet inside, and the Hall effect sensor will generate continuous low-frequency electrical voltage signals due to the nonlinear continuous magnetic flux change.

In this paper, the whisker sensor is used for terrain classification in an unstructured environment, although it is more versatile and could be applied to more demanding application, such as object detection, surface information identification and robot navigation [23].

\section{B. Experimental Setup Design \& Procedure}

The physical reservoir computing system experiment is conducted by a four-wheel mobile robot with the tapered whisker sensor fixed orthogonally on the robot. The wheeled unit and electronic system connection are shown in Fig. 2.

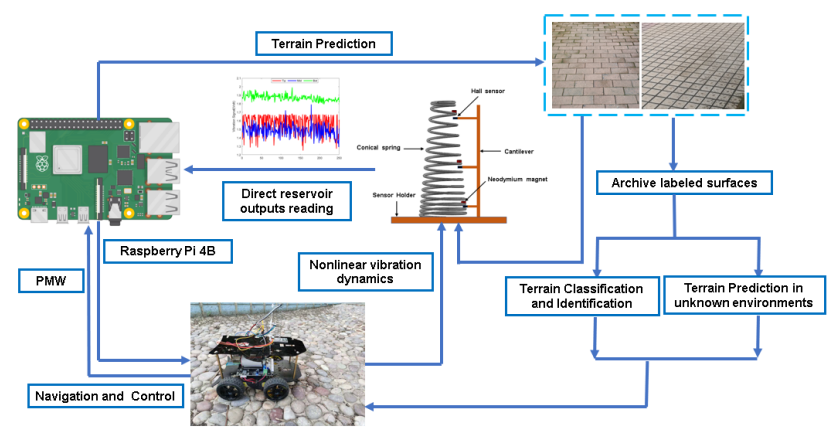

Fig. 3. Diagram of the overall system for the mobile whiskered robot terrain identification, based on the tapered whisker sensor.

Fig.3 shows the computation framework based on reservoir computing systems for the mobile whiskered robot terrain recognition and unknown surface roughness prediction. The three streams output signals of the Hall sensors are sent to a Raspberry Pi 4B via an ADS1115 analog to digital converter as shown in Fig.2. The operating frequency of the AD converter is between $8 \mathrm{~Hz}$ to $860 \mathrm{~Hz}$, which enables the robot to capture enough information even during a quite short time window. The sensor data collection, robot motion and control, reservoir computing, terrain identification and the surface roughness prediction in unknown environments are synchronized and conducted online by the Raspberry Pi 4B because it only requires little computing power.

To extract the reservoir feature vector $R_{f}, n$ sequential vibration voltage points are sampled from the Hall sensors at the top (T), middle (M), and bottom (B) of the tapered spring. The $j$ th data point is denoted as $d_{j}^{T}, d_{j}^{M}, d_{j}^{\mathrm{B}}, j=1,2,3, \cdots, n$ for top, middle, and bottom respectively. A sampling frequency of $f_{\text {samp }}=100 \mathrm{~Hz}$ was used. Sampled data were then grouped into $n \times 3$ sampling segmented vector $D^{n \times 3}$. The $j$ th row of $D^{n \times 3}$ is given by

$$
D_{j}^{1 \times 3}=\left[\begin{array}{lll}
d_{j}^{T} & d_{j}^{M} & d_{j}^{B}
\end{array}\right] \quad j=1,2 \ldots n,
$$

where each matrix row corresponds to the three streams of Hall effect sensor voltage outputs at a given time.

Reservoir computing needs a high dimensional state space with a large readout, but we simplify the readout and choose a limited readout with 3 here from a larger reservoir occurring in the tapered spring to suit the application of terrain classification in this paper. Actually, we could have an infinite number of readouts from the whisker sensor for terrain identification.

\section{METHODOLOGY}

\section{A. Reservoir feature for terrain identification}

A dynamical tapered whisker could provide morphological computation power for projecting features from the external temporal vibration data for model training directly. The external oscillation is fed to the nonlinear whisker reservoir, which is internally composed of random and fixed nonlinear nodes. It only requires the output weight matrix $W_{\text {out }}^{T}$ that connects the reservoir state $x(t)$ and the output $y(t)$ to be trained quickly.

The covariance matrix $C_{D}=\operatorname{cov}_{p, q} \quad p, q=T, B, M$ of the segmented vibration vector $D_{i}^{n \times 3}$ was calculated to enable future exploration of the data dispersion degree and spatial distribution trend.

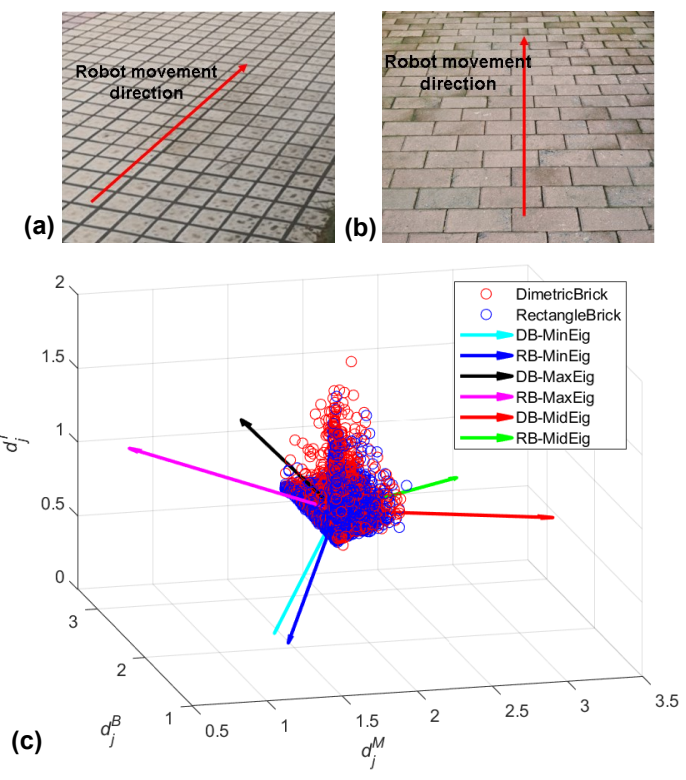

Fig. 4. The three streams Hall effect sensor voltage outputs $d_{j}^{T}, d_{j}^{M}, d_{j}^{B}$ of two similar terrain surface along with the three associated Eigenvector directions and Eigenvalue scaling $(\mathrm{RB}$ represents Rangle Brick and DB represents Dimetric Brick). This demonstrates the advantages of TWRC system that it can discriminate even two very similar terrain surfaces based on the whisker reservoir outputs.

This symmetric matrix is then decomposed into its Eigen representation to extract the eigenvector feature $R_{v e c}$ and eigenvalue features $R_{v a l}$ for subsequent fast logistic regression training.

$$
C_{D}=R_{v e c} R_{v a l} R_{v e c}^{T}
$$


Here, $R_{v e c}$ and $R_{v a l}$ are $3 \times 3$ metrics. The eigenvector $R_{v e c}$ of the raw data's covariance matrix reflects the dispersion direction of the distribution transformation of the sampled data, and the eigenvalue $R_{v a l}$ corresponding to the square of the scaling factor in each direction. Finally, the eigenvector and the eigenvalue of the covariance matrix jointly work as the input for logistic regression training and terrain classification. The dimension of the combined reservoir feature vector $R_{f}$ is $12 \times 1$.

$$
R_{f}=\left[\begin{array}{ll}
R_{v e c} & R_{v a l}
\end{array}\right]^{T}
$$

The tapered whisker-based RC system has excellent discrimination capability, even for very similar external terrain stimulus, e.g. the rectangular and diametric bricks shown in Fig.4 (a) and Fig.4 (b). We can see from Fig.4 that the original vibration data of the two terrains have very similar distributions in the time domain, which makes the distinction very difficult for mobile robots. However, due to the use of the tapered spring, the covariance matrix $C_{D}$ of their original data has distinctly different eigenvalues $R_{v e c}$ and eigenvectors $R_{v a l}$ in Eigen space, as shown in Fig.4 (c). This indicates that this conical spring whisker has good discrimination capabilities for terrain surface identification and it could separate the different frequency vibrations of raw data in the time domain directly.

\section{B. Readout weights training via Logistic Regression}

A simple supervised learning algorithm, one-versus-rest (OVR) logistic regression (LR), was applied to train the linear $\mathrm{RC}$ readout weights to classify different terrains as shown in Fig. 1. Compared with other artificial neural networks, e.g. naive Bayes, cluster, k-nearest neighbors, support vector machine, and convolutional neural networks, logistic regression is a computationally economical classification method based on the theoretical computational complexity.

The input of $\mathrm{RC}$ is the reservoir feature vector $R_{f}$, which is described by a feature vector including 12 elements (the eigenvector $R_{v e c}$ and the eigenvalue $R_{v a l}$ of the covariance matrix). It will be imported into the logistic regression network directly, and the highest probability of the reservoir state corresponding to the expected possible terrain surfaces is determined both by the input reservoir feature vector $R_{f}$ and the LR weight matrix $W_{\text {out }}$, as shown in Fig.1. The LR has six outputs representing each of the six terrain surfaces.

Then we use the sigmoid function given by equation 4

$$
g(\alpha)=\frac{1}{1+e^{-\alpha}}
$$

where $\alpha=W_{\text {out }}^{T} \cdot R_{f}$ which results in

$$
h=g\left(W_{\text {out }}^{T} \cdot R_{f}\right)
$$

The cost function is given by:

$$
\left.\left.J=-\frac{1}{m} \sum_{i=1}^{m}[\alpha \log (h))+(1-\alpha) \log (1-h)\right)\right]
$$

where $m$ is the number of training feature vectors, and $\alpha$ is expected output for the reservoir feature vector $R_{f}$.
The loss function of LR is convex, so the parameter training that minimizes the loss function can be accomplished by the gradient descent method defined as:

$$
\frac{\partial J}{\partial W_{\text {out }}}=\frac{1}{m} \sum_{i=1}^{m}(h-\alpha) R_{f}
$$

\section{SURFACES ROUGHNESS PREDICTION FOR UNKNOWN TERRAINS}

In this section, we experimentally demonstrate that a tapered whisker-based RC system can provide morphological computing power for surface identification. To achieve the roughness estimation for unknown terrains, a novelty detector based on the reservoir features is proposed.

\section{A. Data collection}

An important task for evaluating the performance of our TWRC system is surface selection. Six representative types of surfaces with different hardness and roughness were sampled in our experiments to test the performance of our TWRC system as well as its capability in predicting the roughness of the new unknown terrain surfaces. Two of these were indoor surfaces and four were outdoor terrains:

(1). hard rough cobblestones, (2). hard roughish brick

(3). soft rough grass, (4). soft roughish sand

(5). hard smooth flat (Indoor), (6). soft smooth carpet (Indoor)

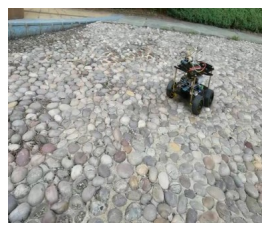

(a)

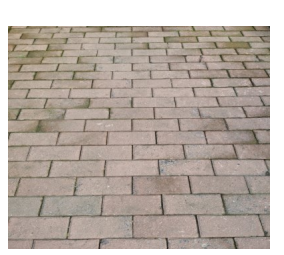

(b)

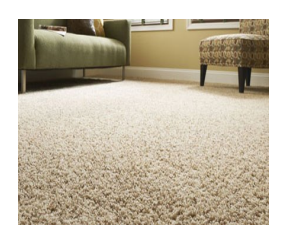

(c)
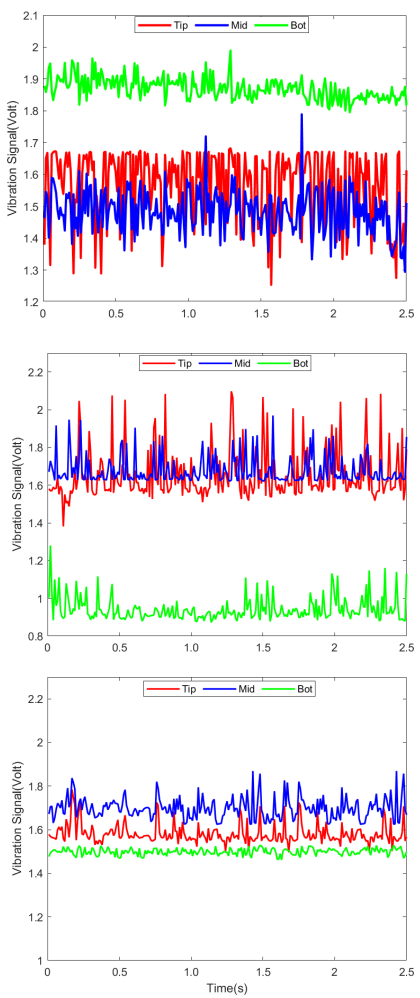

Fig. 5. Three terrain surfaces along with the associated three streams vibration signal, used to evaluate the performance of the RC system. (a) Hard Rough gravel. (b) Hard Roughish Brick. (c) Soft Smooth Carpet.

When the whiskered robot moved at a steady speed of $0.2 \mathrm{~m} / \mathrm{s}$ traversing the different surfaces, the Raspberry Pi $4 \mathrm{~B}$ will automatically collect the vibration data via the $A / D$ 
converter ADS1115 with a sampling frequency of $100 \mathrm{~Hz}$, and label the raw vibration data. Then, the collected data was segmented into single training feature vectors with different time window $T=1.5$ or 3 seconds for later procession. In total, 750 seconds of vibration data of our system were collected for each terrain surface, which finally led to the construction of our dataset $\mathbf{D}_{i}^{n \times 3}$ for feature extraction and reservoir logistic regression training. Part of the sampled experiment terrain surface and its corresponding three streams vibration data are given in Fig.5.

\section{B. Mahalanobis distance in the eigen space}

Based on the aforementioned reservoir feature extraction methods and vibration dataset, the reservoir features including the eigenvectors and eigenvalues, the RC system was calculated with cost-efficient computation power. Fig. 6 demonstrated the eigenvalue feature distribution of the conical whisker reservoir outputs for six terrain surfaces in the Eigen space.

As can be seen from Fig.6, external surface stimuli of different hardness and roughness terrains will result in unique eigenvalues outputs for our whisker-based RC system. For example, the roughness of the gravel terrain results in a relatively large eigenvalues compared with other surfaces due to its coarse gravel. In contrast, when the whiskered robot operated at a steady speed over the flat and carpet, the eigenvalues of the conical whisker reservoirs outputs were mainly distributed near the zero points and have a relatively smaller dispersion. Even though the actual topographic features of carpet and float are very similar, their eigenvalue distribution in feature space is also completely different. This indicates that based on the already calibrated TWRC system, the robot moving in steady-state motion could autonomously predict the roughness or softness even of very similar terrain, based on prior knowledge.

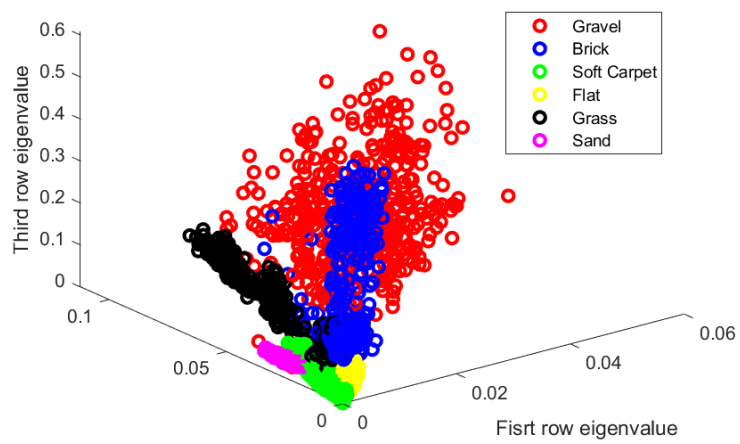

Second row eigenvalue

Fig. 6. Eigenvalues of the tapered whisker reservoir outputs for six sampled terrain surfaces in Eigen space for terrain classification.

In order to achieve roughness prediction for unknown new terrain, we introduce a Mahalanobis distance $R_{\mathrm{D}}$ in the Eigen space. The distance can reflect the correlation between the reservoir features $\hat{\mathbf{R}}_{f}$ of unknown terrain and the existing data reserve feature base $\mathbf{R}_{f}$ in Eigen space. It can be expressed mathematically as

$$
R_{\mathrm{D}}=\sqrt{\left(\hat{\mathbf{R}}_{f}-\mathbf{R}_{f}\right)^{T} C_{D}^{-1}\left(\hat{\mathbf{R}}_{f}-\mathbf{R}_{f}\right)}
$$

The metric $R_{\mathrm{D}}$ is scale-invariant and could eliminate the effect of variance differences caused by the distribution of samples in different dimensions, which is important for distinguishing similar terrain surfaces. When this value is smaller in equation 8 , it indicates that the new data has a higher probability of belonging to a particular class dataset. It especially considers the interaction between the eigenvector's direction and eigenvalues and their combined influence on the final distance $R_{\mathrm{D}}$ because these features together reflect the textural characteristics of the terrain.

To verify that the proposed distance $R_{\mathrm{D}}$ is a good characteristic criteria for predicting unknown terrain, we calculated the distances $R_{\mathrm{D}}$ between the validation testing dataset $\mathbf{D}_{t}$ and the prior dataset $\mathbf{D}_{p} .70 \%$ of the raw data $\mathbf{D}^{n \times 3}$ of each terrain were randomly selected as prior knowledge dataset $\mathbf{D}_{p}$, and the remaining $30 \%$ were used as the testing dataset $\mathbf{D}_{t}$.
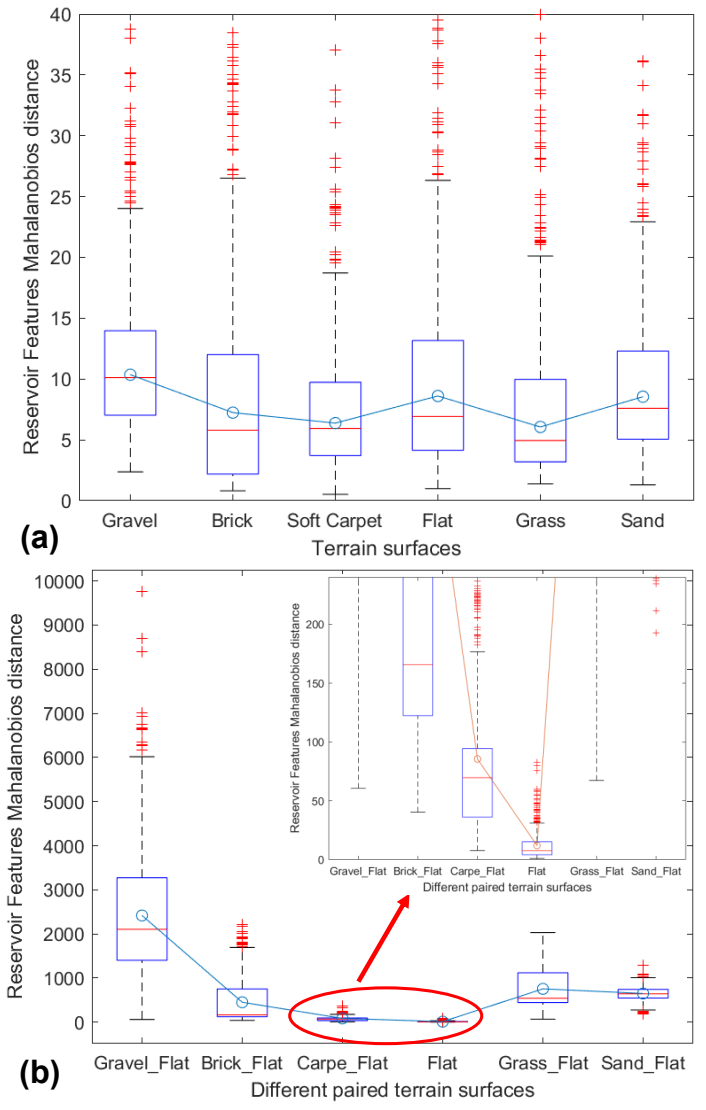

Fig. 7. Boxplot of the Mahalanobis distance in the Eigen space $R_{\mathrm{D}}$ distribution based on the reservoir features $\mathbf{R}_{f}$. The red cross points represent the outliers. (a) The $R_{\mathrm{D}}$ between the validation dataset $\mathbf{D}_{t}$ relative to the a priori dataset $\mathbf{D}_{p}$ for each terrain. (b) The $R_{\mathrm{D}}$ between the flat terrain dataset relative to the other remaining five terrains dataset.

The distribution of the $R_{\mathrm{D}}$ between the validation feature dataset $\mathbf{D}_{t}$ and the corresponding terrain prior information set $\mathbf{D}_{p}$ is given in Fig.7 (a). At the same time, we calculated the distance between the flat relative to the other five terrain 
surfaces, and the distribution of the distance results $R_{\mathrm{D}}$ is shown in Fig.7 (b). In this test, 5 random experiments were conducted to avoid accidental distance calculation errors caused by random trials.

In Fig.7 (a), it can be seen that for all six terrain surfaces, the average Mahalanobis distance (AMD) $R_{\mathrm{D}}$ between the testing dataset $\mathbf{D}_{t}$ and the prior dataset $\mathbf{D}_{p}$ is 7.87 , with a average standard deviation (ASD) of 4.94. For each terrain there are some anomalous distance values, which can be explained by the eigenvalues of those marginal zones between different terrains, as shown in Fig.6. However, for each terrain, almost all of the data excluding outliers are distributed within about (AMD+3ASD) 22.69 .

Combining the results of the distance distribution given in Fig.7, we can find that if a set of feature data does not belong to the particular corresponding dataset, then the value of $R_{\mathrm{D}}$ will be significantly larger. For example, the average value $R_{\mathrm{D}}$ between the flat testset and the gravel prior database reaches 2354, while the average distance between the flat testset and its corresponding priori database is only 8.6. Although the flat and the carpet are smooth and very similar terrains, we can also distinguish them with a high strict distance boundary condition.

However, this also means that we cannot predict the unknown terrain efficiently if we rely only on the distance $R_{\mathrm{D}}$. Therefore, a model combining the discrimination distance $R_{\mathrm{D}}$ with linear output training is proposed in the next subsection.

\section{Surfaces roughness prediction for unknown terrains}

In this section, we will describe in detail how to predict the roughness of an unknown terrain based on the extendedTWRC system including the novelty detector. The physical system working flow framework is shown in Fig.8.

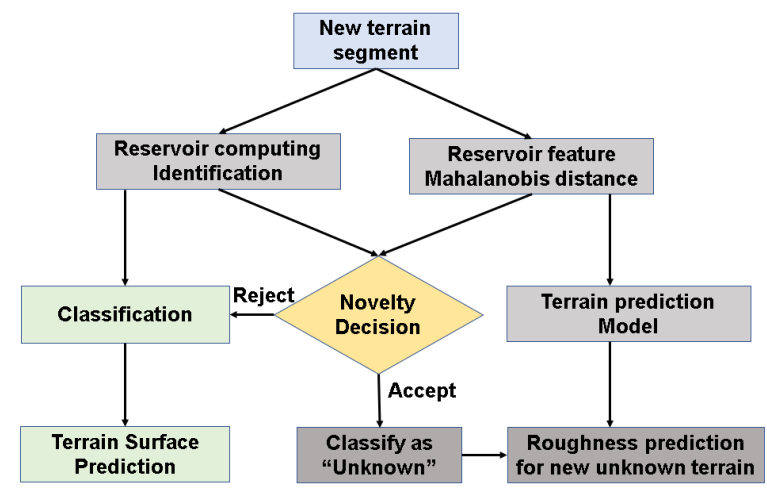

Fig. 8. Workflow of the roughness estimation of unknown terrain surface based on the extended-TWRC system including the novelty detector.

As shown in Fig.8, this prediction process starts with the collection of a new terrain segment $D^{\text {new }}$ and the computation of its corresponding reservoir feature vector $R_{f}^{n e w}$. In the next step, the novelty decision detector will determine whether this new terrain is a known terrain (rejected) or an unknown terrain (accepted) based on the trained logistic regression model and the distribution of reservoir features of the prior known terrain. In case of reject, the mobile robot will identify the new segment's terrain type using reservoir computing based on the trained logistic regression weights with low computation cost.

If the new terrain segment $D^{n e w}$ is "accepted", then the robot can expeditiously predict the surface properties roughness of this new terrain based on the labeled prior-terrain information and the discrimination distance $R_{\mathrm{D}}$. The focus of this paper is to show that our proposed method can make a reasonable estimate of the roughness of unknown terrain rather than giving very detailed roughness information, as it relies heavily on previously calibrated terrain roughness information.

\section{RESULTS}

To verify the reliability and robustness of the aforementioned methods, surface identification experiments based on the tapered whisker-based reservoir computing system were conducted on six different terrains by the mobile robot at a steady traverse speed. Comparative experiments were conducted to demonstrate that it is not sufficient to do classification work if linear IMU data, e.g., acceleration, are used directly. Subsequently, we tested the performance of our extended-TWRC for estimating the unknown terrain's roughness based on previously calibrated terrains.

\section{A. Reservoir computing classification results}

In this section, we evaluate the performance of our physical reservoir computing system based on the aforementioned feature extraction methods because these could be calculated at the same time without extra computing power. The sampling dataset was segmented into raw terrain feature vectors $D^{n \times 3}$ corresponding to different time windows $T=1.5 \mathrm{~s}$ and $3 \mathrm{~s}$ to test the performance of our proposed TWRC system.
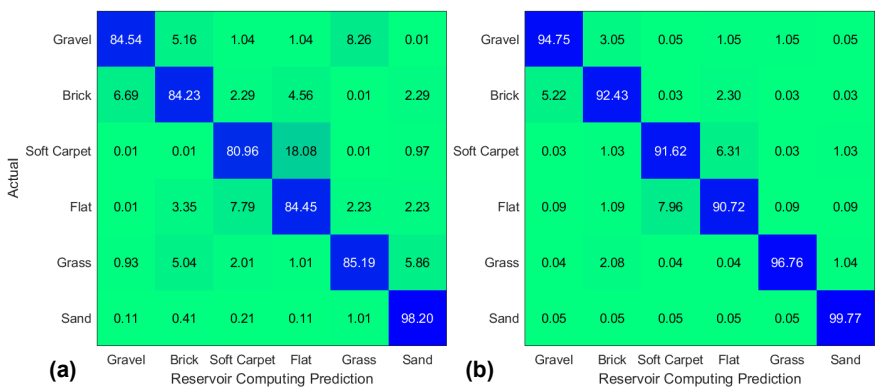

Fig. 9. Confusion matrix of reservoir computing prediction success rate averaged over 20 randomly trials, with robot speed is $0.2 \mathrm{~m} / \mathrm{s}$ and the sampling frequency of the whisker is $100 \mathrm{~Hz}$. (a) Prediction results for time window size $1.5 \mathrm{~s}$. (b) Prediction results for time window size $3 \mathrm{~s}$.

During the repeated random subsampling validation experiments, $75 \%$ of data $\mathbf{D}_{\text {train }}^{n \times 3}$ were randomly selected for the LR weight matrix training, and the remaining $25 \%$ of data $\mathbf{D}_{\text {test }}^{n \times 3}$ were used to test the performance of our proposed reservoir computing system. In total, 20 random experiments were conducted to avoid accidental classification errors caused by random trials.

Fig. 9 (a) and (b) show the prediction confusion matrix of the reservoir computing system for 20 random experiments when the whiskered robot moved at a steady speed at $0.2 \mathrm{~m} / \mathrm{s}$, and the A/D converter sampling frequency is $100 \mathrm{~Hz}$. The 
average prediction rate of these test experiments are $86.2 \%$ and $94.3 \%$ for the time windows $1.5 \mathrm{~s}$ and $3 \mathrm{~s}$ respectively. The results demonstrate that our TWRC system has good recognition and identification capabilities of different terrain surfaces when the whiskered robot executes robust real-world deployments action. Even though longer time windows could augment classification accuracy as shown in Fig. 9 (a) and (b). However, a trade-off is needed between real-time constraints and identification accuracy.

There is still about $7 \%$ confusion between the soft carpet and flat ground even if the time window is 3 seconds. This is because these two indoor terrains are quite similar and the reservoir cannot map enough information over short time windows for classification. However, these two indoor surfaces have low confusion values with the outdoor terrains because their eigenfeatures were quite distinguished. The sand achieves the best performance with $98.2 \%$ during the experiments, which even had no false positives. In the case of the brick terrains, it has a complicated surface such as joint gaps, and a rugged surface which makes the conical whisker sensor more challenging to distinguish it from gravel and flat terrains. A more advanced classifier might make progress when it is applied to the test compared with the logistic regression,but this goes beyond the focus of this paper.

An IMU was mounted on the mobile robot to collect vertical acceleration signals in six terrains at a steady speed at $0.2 \mathrm{~m} / \mathrm{s}$ with same sampling frequency $100 \mathrm{~Hz}$. The acceleration data was segmented into vectors with time window $1.5 \mathrm{~s}$ to train the same above logistic regression model directly and classify the different six terrains. Table.I shows the classification results of different time windows. These results indicate that it cannot achieve terrain classification by directly using linear acceleration output. To achieve terrain classification with high accuracy, the linear acceleration data requires training a complex neural network after extracting multiple time-domain features as shown in [10], which requires more computational resources than our reservoir computing approach.

TABLE I

TERRAINS CLASSIFICATION ACCURACY USING ACCELERATION DATA TRAIN THE LOGISTIC REGRESSION NETWORK DIRECTLY

\begin{tabular}{ccc}
\hline & Time Window 1.5s & Time Window 3s \\
\hline $\begin{array}{c}\text { Prediction accuracy using } \\
\text { acceleration data }\end{array}$ & $14.9 \%$ & $15.2 \%$ \\
\hline
\end{tabular}

\section{B. Surface roughness estimation of unknown terrains}

The results in the previous section have shown that the TWRC system has good classification and identification capabilities on the six presented terrain surfaces when the robot is moving at a steady speed. However, without the novelty detection, the new unknown terrain signals would be classified wrongly which might lead to adverse consequences for the robots. In this section, we will test our proposed system's performance for predicting the unknown surfaces' properties based on prior reservoir information.

To verify the distinctive capability of the extended TWRC system, we use the soft carpet terrain as the testset $\mathbf{D}_{t}$ to represent the unknown terrain, because of its close resemblance to the flat terrain which might lead to difficulty in identifying the outliers and better reflecting the superiority of our prediction strategy. The remaining 5 terrains will be used as a priori datasets $\mathbf{D}_{p}$ to train the reservoir computing model and novelty detection and prediction strategy. We manually calibrated the roughness of these 5 terrains which increases from flat to gravel in order to predict the roughness of the unknown terrain. In Fig.11, the coordinates of the Y-axis indicates different terrains whose roughness increases in order from bottom to top. The Y-axis spacing does not accurately reflect their roughness, since the purpose of this is simply to facilitate the demonstration that our system can roughly predict the terrain's roughness based on prior information. The closer spacing of unknown terrain to a particular terrain only indicates that they have more similar roughness to each other.

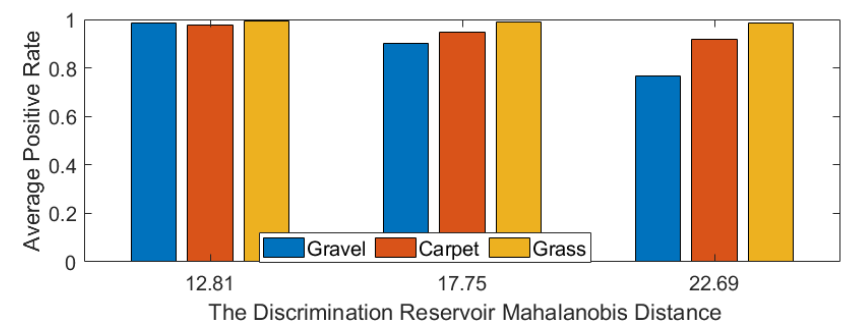

Fig. 10. The novelty positive rate of different unknown testing terrain datasets. Experiments are done on three different discrimination distance $R_{\mathrm{D}}$ of these three testing terrains.

Different terrains were selected as unknown terrain test sets $\mathbf{D}_{t}$ to test the performance of our novelty detectors. Fig.10 demonstrated the novelty positive rate for different testing samples at different distance $R_{\mathrm{D}}$. It can be seen that the accuracy of outlier detection decreases as the discrimination distance $R_{\mathrm{D}}$ decreases, and its average positive rate decreases from $97.51 \%$ at 12.81 to $89.1 \%$ at 22.69 . This means that in order to improve security, the strict discrimination distance $R_{\mathrm{D}}$ must be adopted by mobile robots.

Fig.11 showed the 300 prediction results of the actual and predicted terrain types based on the above methods, with carpet working as the testing unknown dataset $\mathbf{D}_{t}$. The whiskered robot could very accurately identify the new unknown terrain $\mathbf{D}_{t}$ and discriminate it from the already existing terrains inside the priori datasets $\mathbf{D}_{p}$. As shown in Fig.11, it could perform highly accurate terrain identification for the terrain in the prior database $\mathbf{D}_{p}$ based on reservoir computing at the same time. The average classification accuracy reached for known terrain $83.7 \%$, while the detection accuracy for unknown terrain was $88.7 \%$ for the extended TWRC.

More importantly, it achieved the roughness estimate of the unknown terrain, which is approximately between the flat and the sand, as shown in Fig.11. However, the roughness of the unknown terrain is more similar to the floor terrain because it is located closer to the flat on the Y-axis. These results demonstrated that our whisker sensor as well as the proposed extended TWRC system can identify the unknown terrain well and give a roughness estimation of it in general. If a quantified terrain roughness dataset had been given, our extended TWRC 


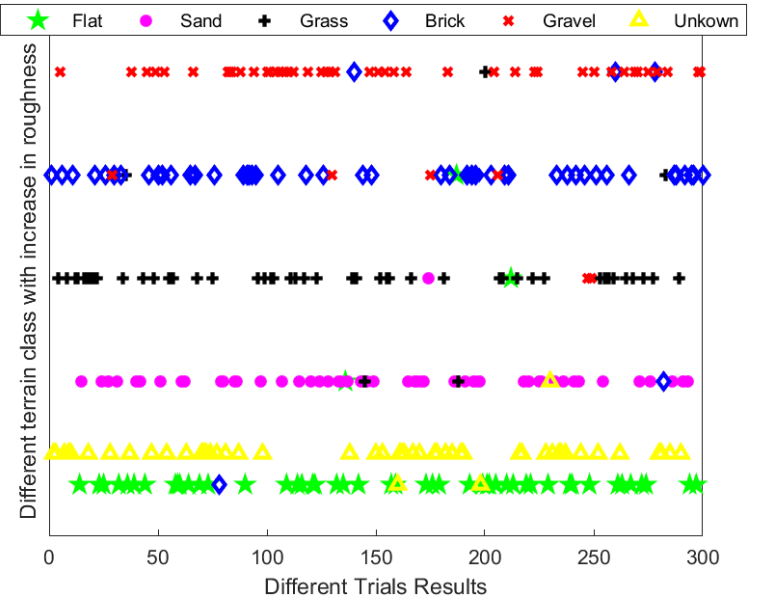

Fig. 11. The roughness prediction results when carpet terrain was chosen as the unknown terrain surface with the discrimination distance $R_{\mathrm{D}}=12.84$. Y-axis indicates the different terrain classes with a increase of the roughness in order from bottom to top, and X-axis denotes the prediction results of 300 trials. The roughness of the unknown terrain is more similar to the flat terrain because it is located closer to the flat terrain on the Y-axis.

system could quantify and accurately predict the roughness of unknown terrain based on the prior datasets.

\section{CONCLUSIONS}

Addressing cost-efficient terrain classification and roughness estimation of unknown terrain in uncalibrated environments, we proposed a solution employing a tapered whiskerbased physical reservoir computing (TWRC) system with limited signal readouts for terrain classification. We also proposed an extended TWRC system for roughness estimation of unknown terrains. Experimental results from the proposed reservoir computing system show that the compliant nonlinear whisker sensor could achieve frequency separation in the time domain directly even only with a limited number of Hall sensor readings and without any data processing before model training. The results show that the steady response of an tapered whisker sensor could be used to identify the terrain texture even between very similar terrains, and that a mobile robot equipped with an extended TWRC system could estimate the unknown terrain's roughness based on the prior reservoir information.

In the future, the reliability and robustness testing experiments in more complex and unknown scenarios will be worth exploring. It would also be of interest to investigate how stiffness control of the tapered whisker can be used as a control parameter to improve its identification performance.

\section{REFERENCES}

[1] R. Siegwart, I. R. Nourbakhsh, and D. Scaramuzza, Introduction to autonomous mobile robots. MIT press, 2011.

[2] A. Howard and H. Seraji, "Vision-based terrain characterization and traversability assessment," Journal of Robotic Systems, vol. 18, no. 10, pp. 577-587, 2001.

[3] B. Suger, B. Steder, and W. Burgard, "Traversability analysis for mobile robots in outdoor environments: A semi-supervised learning approach based on 3d-lidar data," in 2015 IEEE International Conference on Robotics and Automation (ICRA). IEEE, 2015, pp. 3941-3946.
[4] A. Valada and W. Burgard, "Deep spatiotemporal models for robust proprioceptive terrain classification," The International Journal of Robotics Research, vol. 36, no. 13-14, pp. 1521-1539, 2017.

[5] C. A. Brooks and K. Iagnemma, "Vibration-based terrain classification for planetary exploration rovers," IEEE Transactions on Robotics, vol. 21, no. 6, pp. 1185-1191, 2005.

[6] M. Fend, S. Bovet, H. Yokoi, and R. Pfeifer, "An active artificial whisker array for texture discrimination," in Proceedings 2003 IEEE/RSJ International Conference on Intelligent Robots and Systems (IROS 2003)(Cat. No. 03CH37453), vol. 2. IEEE, 2003, pp. 1044-1049.

[7] J. Zürn, W. Burgard, and A. Valada, "Self-supervised visual terrain classification from unsupervised acoustic feature learning," IEEE Transactions on Robotics, vol. 37, no. 2, pp. 466-481, 2020.

[8] L. Wellhausen, A. Dosovitskiy, R. Ranftl, K. Walas, C. Cadena, and M. Hutter, "Where should i walk? predicting terrain properties from images via self-supervised learning," IEEE Robotics and Automation Letters, vol. 4, no. 2, pp. 1509-1516, 2019.

[9] E. M. Dupont, C. A. Moore, E. G. Collins, and E. Coyle, "Frequency response method for terrain classification in autonomous ground vehicles," Autonomous Robots, vol. 24, no. 4, pp. 337-347, 2008.

[10] P. Giguere and G. Dudek, "A simple tactile probe for surface identification by mobile robots," IEEE Transactions on Robotics, vol. 27, no. 3, pp. 534-544, 2011.

[11] M. Salman and M. J. Pearson, "Advancing whisker based navigation through the implementation of bio-inspired whisking strategies," in 2016 IEEE International Conference on Robotics and Biomimetics (ROBIO). IEEE, 2016, pp. 767-773.

[12] T. J. Prescott, M. J. Pearson, B. Mitchinson, J. C. W. Sullivan, and A. G. Pipe, "Whisking with robots," IEEE robotics \& automation magazine, vol. 16, no. 3, pp. 42-50, 2009.

[13] Z. Yu, S. Sadati, H. Wegiriya, P. Childs, and T. Nanayakkara, "A method to use nonlinear dynamics in a whisker sensor for terrain identification by mobile robots," arXiv preprint arXiv:2108.02267, 2021.

[14] M. Salman and M. J. Pearson, "Whisker-ratslam applied to 6d object identification and spatial localisation," in Conference on Biomimetic and Biohybrid Systems. Springer, 2018, pp. 403-414.

[15] M. Brecht, B. Preilowski, and M. M. Merzenich, "Functional architecture of the mystacial vibrissae," Behavioural brain research, vol. 84, no. 1-2, pp. 81-97, 1997.

[16] M. J. Pearson and M. Salman, "Active whisker placement and exploration for rapid object recognition," in 2019 IEEE/RSJ International Conference on Intelligent Robots and Systems (IROS). IEEE, 2019, pp. 672-677.

[17] M. Kaneko, N. Kanayama, and T. Tsuji, "Active antenna for contact sensing," IEEE Transactions on robotics and automation, vol. 14, no. 2 , pp. 278-291, 1998.

[18] H. Hauser, "Physical Reservoir Computing in Robotics," Natural Computing Series, pp. 169-190, 2021. [Online]. Available: https: //link.springer.com/chapter/10.1007/978-981-13-1687-6_8

[19] M. Lukoševičius, H. Jaeger, and B. Schrauwen, "Reservoir computing trends," KI-Künstliche Intelligenz, vol. 26, no. 4, pp. 365-371, 2012.

[20] K. Nakajima, "Physical reservoir computing — an introductory perspective," Japanese Journal of Applied Physics, vol. 59, no. 6, p. 060501, 2020.

[21] K. Nakajima, H. Hauser, T. Li, and R. Pfeifer, "Information processing via physical soft body," Scientific reports, vol. 5, no. 1, pp. 1-11, 2015.

[22] H. Hauser, A. J. Ijspeert, R. M. Füchslin, R. Pfeifer, and W. Maass, "Towards a theoretical foundation for morphological computation with compliant bodies," Biological cybernetics, vol. 105, no. 5, pp. 355-370, 2011.

[23] H. K. Tripathy, S. Mishra, H. K. Thakkar, and D. Rai, "Care: A collisionaware mobile robot navigation in grid environment using improved breadth first search," Computers \& Electrical Engineering, vol. 94, p. 107327,2021 